\title{
Gut Microbiota Profile Autism Spectrum Disorder Relationship: Diversity and Imbalance in Probiotics
}

\author{
Aurelie Razafindralambo and Hary Razafindralambo* \\ University of Liege, Gembloux Agro-Bio Tech, TERRA Teaching and Research Centre Avenue de la Faculty 2B, BAT 140, B-5030 \\ Gembloux, Belgium
}

\begin{abstract}
Probiotic-based diet supplementations are among potential treatments of Autism Spectrum Disorders (ASDs) because of the existing gut microbiota profile-mental health. In order to conceive a personalized probiotic supplement to a specified autistic adult, the fecal microbiota of his neurotypical father, mother and sister, have been analyzed and compared. The latest $16 \mathrm{~S}$ rRNA technology was used for microbial determination at species level (Mymicrozoo analysis, The Netherlands). Less microbiota diversity and an uncommon higher Streptococcus/Lactobacillus abundance ratio were revealed in the autistic adult compared to his relatives. These results were discussed in relation to his digestive issues.
\end{abstract}

Keywords: Diet supplementation; ASD; Gluten intolerance; Digestive troubles; Streptococcus; Lactobacillus ratio

\section{INTRODUCTION}

Autism spectrum disorder (ASD) is a range of neurodevelopmental disorders which are characterized by impairment in social behavior, communication and language, and restrictive and repetitive behaviors. Given the dramatic rise of its prevalence over the last few years, ASD is considered as epidemic according to the World Health Organization (WHO) estimation-There exists now 1 in 160 children with ASD, whereas 2 to 4 cases per 10,000 children were diagnosed in the 1970s [1].

Although genetic alterations are the most well-known risk factors of ASD [2], other possible causes from impairment of the gut function have also been frequently reported $[2,3]$. ASD patients often suffer from other comorbitities, including epilepsy, immune dysfunction, and digestive troubles such as constipation, diarrhea, and food intolerance [4].

Different dietary strategies, mainly restrictive diets and food supplements, have been suggested to improve the conditions of ASD patients. Assuming the relationship between the gut and mental health [5], probiotics have been proposed as potential food supplements for improving the well-being of individuals with ASD. By definition, probiotics are live micro-organisms which, if administrated in adequate amount, convey a positive effect on the host [6]. Probiotics consumption boosts not only the immune and digestive health but also the central nervous system function in both animals and humans [7]. Moreover, the current trends draw up the potentiality of probiotics in improving human life and solving the most devastator diseases of the 21 st century by offering natural, safe, and cost-effective solutions [8].

In a recent case report, we have shown the success of nutritherapy for solving digestive troubles in a gluten intolerant autistic adult living with his parents and sister [9]. In this case, the autism is mainly characterized by impairment in communication and language with no real signs of social interaction troubles, while being of non-genetic origin, regarding the absence of autistic genes mutation in the patient's DNA (CNTNAP2 and NRXN1). Recently, we have reviewed the possible link between gluten intolerance and ASD, and discussed innovative routes based on probiotic supplement diets for managing ASD [10].

In order to get some insight into the relationship between such ASD case and gut microbiota composition, the fecal bacteria of all family members have been analyzed and compared. Our objective particularly aims to establish probiotic profiles, and provides subsequently possible personalized probiotic

"Correspondence to: Hary Razafindralambo, University of Liege, Gembloux Agro-Bio Tech, TERRA Teaching and Research Centre Avenue de la Faculty 2B, BAT 140, B-5030 Gembloux, Belgium, Tel: +3281622148; E-mail: h.razafindralambo@uliege.be

Received: June 18, 2019; Accepted: July 08, 2019; Published: July 15, 2019

Citation: Razafindralambo A, Razafindralambo H. (2019) Gut Microbiota Profile Autism Spectrum Disorder Relationship: Diversity and Imbalance in Probiotics. J Prob Health. 7:209.

Copyright: (C) 2019, Razafindralambo A, et al. This is an open-access article distributed under the terms of the Creative Commons Attribution License, which permits unrestricted use, distribution, and reproduction in any medium, provided the original author and source are credited. 
supplements for improving the digestive and mental health of the ASD patient.

In this short-communication, we report the results of the family's fecal analysis by the latest 16S rRNA technology, pointing out the diversity in microbiota, as well as an uncommon substantially higher Streptococcus/Lactobacillus ratio in the ASD-affected individual compared to his relatives.

\section{MATERIALS AND METHODS}

\section{Population}

A family of four adults who live in the same household and include an individual with ASD was subjected to the fecal microbiota analysis. (Father: 50 years, BMI: 29; Mother: 49 years, BMI: 28; Sister: 22 years, BMI: 18; Son with ASD: 20 years, BMI: 22).

\section{Stool sampling procedure}

The sampling procedure was briefly described as follow. A small amount of feces (approximately $250 \mathrm{mg}$ ) was transferred with a cotton swab to a tube provided by MyMicroZooTM (www.mymicrozoo.com). A volume of stabilizing liquid was added into the tube for preserving the fecal sample composition during transport. The tubes were shipped to the MyMicroZooTM lab for the subsequent steps of the analysis.

\section{Microbiome analysis}

The microbiome analysis of stool samples was performed by MyMicroZooTM, using the 16S rRNA technology procedure. Briefly, the DNA was extracted from the samples, and the $16 \mathrm{~S}$ rRNA genes were amplified using Polymerase Chain Reaction (PCR) in order to obtain enough material for sequencing. The 16S rRNA genes were then sequenced on Illumina MiniSeq for identification and enumeration of the bacteria in each sample. The abundance of different bacteria at the species level in each stool sample was determined by the number of analyzed specific sequences.

\section{RESULTS}

Figure 1 shows an example of results provided by MymicrozooTM after analysis of the fecal samples. This pie

Table 1: Probiotic profiles in fecal samples: diversity index and main probiotic abundances.

\begin{tabular}{|c|c|c|c|c|}
\hline & Father & Mother & Daughter & Son with ASD \\
\hline Diversity index & 6.8 & 7.1 & 6.5 & 6 \\
\hline Bifidobacterium longum & 1 & 2 & 23 & 568 \\
\hline Bifidobacterium adolescentis & 1602 & 626 & 3575 & 464 \\
\hline Bifidobacterium catenulatum & 26 & 68 & 0 & 0 \\
\hline Bifidobacterium dentium & 2 & 0 & 0 & 5 \\
\hline
\end{tabular}

chart indicates the composition in percent of the fecal bacteria of the adult with ASD. Only species abundance superior to $2.7 \%$ are indicated in the legend. Table 1 lists the diversity index of each individual provided by MyMicroZooTM, as well as the abundance of main strains of probiotics found in each fecal sample. The diversity index calculated in this case is the Shannon index. The higher the number of different bacteria detected in the sample and the more they are equally distributed, the higher the diversity index. In the four samples, the diversity indexes range from 6 to 7.1, the ASD-affected individual having the lowest index score. Nevertheless, these values are arbitrary, and only provide a general indication of gut health.

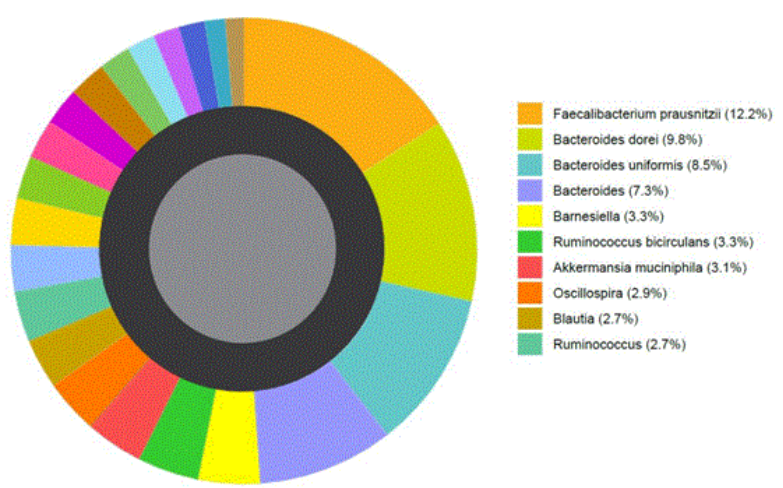

Figure 1: Pie chart provided by Mymicrozoo of the composition of fecal bacteria of the individual with ASD.

The absolute abundance of every bacteria detected in fecal samples has been counted from tablesheet files provided by MymicrozooTM (Supplemental data 1). Main probiotic abundances at the strains level are listed in Table 1 . The values vary greatly from 0 to 3575 . By comparing the total abundance in the three main genera, Bifidobacterium, Lactobacillus and Streptococcus, it appears that Lactobacillus is 3-50 times lower in the ASD-affected person compared to his neurotypical relatives. In contrast, Streptococcus appears to be 10-30 times more abundant in the ASD-affected person compared to those of others. 


\begin{tabular}{|c|c|c|c|c|}
\hline Bifidobacterium kashiwanohense & 3 & 7 & 0 & 0 \\
\hline Bifidobacterium ruminantium & 1 & 2 & 4 & 0 \\
\hline Bifidobacterium total & 1635 & 705 & 3602 & 1037 \\
\hline Lactobacillus plantarum & 20 & 0 & 0 & 16 \\
\hline Lactobacillus rogosae & 35 & 844 & 46 & 0 \\
\hline Lactobacillus ruminis & 17 & 69 & 0 & 0 \\
\hline Lactobacillus helveticus & 0 & 0 & 0 & 1 \\
\hline Lactobacillus johnsonii & 0 & 0 & 0 & 1 \\
\hline Lactobacillus total & 72 & 913 & 46 & 18 \\
\hline Streptococcus salivarius & 7 & 16 & 12 & 52 \\
\hline Streptococcus thermophilus & 24 & 0 & 0 & 338 \\
\hline Steptococcus total & 31 & 16 & 12 & 390 \\
\hline
\end{tabular}

In order to highlight the differences in abundance of probiotics, the three genera abundance ratios plotted in logarithm scale Yaxis are shown in Figure 2. While the Lactobacillus/ Bifidobacteria and Streptococcus/Bifidobacteria ratios are in about the same order of magnitude for each adult, the Streptococcus/Lactobacillus ratio is significantly higher in the ASD-affected person compared to those of his neurotypical relatives.

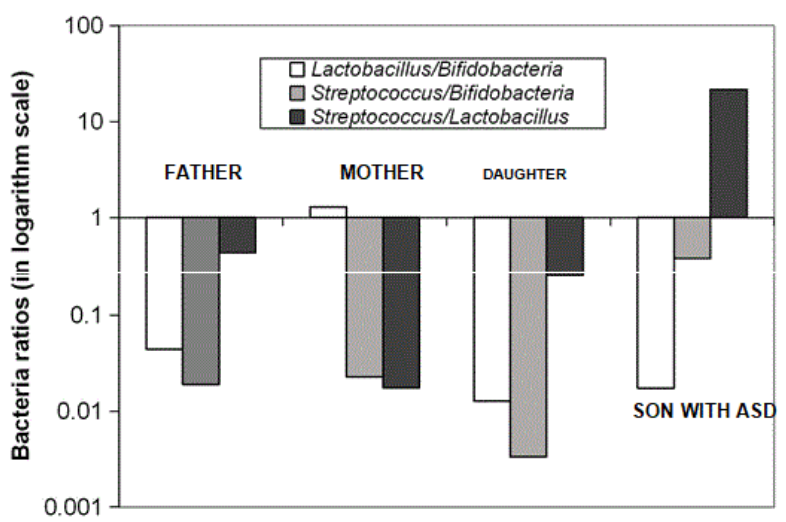

Figure 2: Logarithm of the ratios of different type of bacteria for the four family members.

\section{DISCUSSION}

As a spectrum of disorder, ASD covers a range of conditions. Thus, its symptoms may vary from one individual to another. In the current investigation, the concerned ASD-affected person is a young adult carrying no mutated genes responsible for the main risk of autism (CNTNAP2 and NRXN1), according to a DNA sample analysis (Supplemental data 2). Additionally, no reluctance to social interactions but rather impairment in communication and language characterizes his ASD symptoms.
On the other hand, a 24-hour electroencephalogram analysis revealed no epilepsy sign (Supplemental data 3). By contrast, he was suffering from digestive troubles, primarily due to gluten intolerance, gastroesophageal reflux disease (GERD), and stomach ulcers(9). Such information led us to focus on his gut health as the main potential factor of his ASD. In fact, the fecal microbiota of ASD group is generally shown dissimilar compared to those of normal children[11,12]. Interestingly, our results show differences in the ASD-affected individual's fecal microbiota compared to those of his family member, who live in the same household and follow almost the same diet. His particular stomach conditions (GERD, ulcers), as well as the presence of an important level of heavy metals $(\mathrm{Hg}$, Bs, As) in his body (Supplemental data 4) appear as possible causes of the relative lower microbiota diversity, and the 50 to 1000 times higher ratio of Streptococcus/Lactobacillus in his case. On one hand, it is assumed that digestive environmental conditions are particularly more toxic to the most sensitive probiotics Lactobacillus sp.[13]. This therefore impacts their viability in the gut, and lower their amount in the ASD-affected individual feces. On the other hand, the large amount of Streptococcus in his fecal sample could be related to the autistic individual gluten intolerance. In fact, mucosal damages in the small intestinal might compromise the adhesion of Streptococcus, known as probiotic genera with the lowest surface cell hydrophobicity among microorganisms [14]. By assuming both facts, that is, with lower adhesion capacity and host-mucosal damages, Streptococcus genera can detach easily from the gut epithelium surface, leading to a significant higher abundance of these bacteria in the individual's feces with ASD. Another possible cause arises from the lower microbiota diversity which would have a positive impact on the colonization and abundance of Streptococcus species in his gut. 


\section{CONCLUSION}

Microbiota analyses of ASD-affected and neurotypical family members reveal qualitative and quantitative differences in their gut probiotic composition. First, the diversity of the autistic microbiota is slightly lower than those of his relatives. Second, the Streptococcus-Lactobacillus ratio is noticeably higher in the autistic adult compared to the others. Interestingly, Streptococcus genera appear to be substantially abundance in this case, and could serve as an interesting indicator for specified ASD symptoms. Further research works are needed for investigating the gut probiotic composition-ASD relationship, and especially for better understanding the mechanisms involved in such microbiota alteration. Besides, nutritherapy based on personalized probiotics supplements can be envisaged in order to restore the observed gut microbiota imbalance within autistic individuals.

\section{ACKNOWLEDGMENTS}

This research received no specific grant from any funding agency, commercial or not-for-profit sectors.

\section{CONFLICT OF INTEREST}

All authors declare no conflicts of interest in this paper.

\section{REFERENCES}

1. Boat TF, Wu J. Mental disorders and disabilities among lowincome children. National Academies Press, USA. (2015).

2. Emberti GL, Mazzone L, Benvenuto A, Fasano A, Alcon AG. Risk and protective environmental factors associated with autism spectrum disorder: Evidence-based principles and recommendations. J Clin Med. 2019;8(2):217.

3. Berding K, Donovan SM. Microbiome and nutrition in autism spectrum disorder: Current knowledge and research needs. Nutr Rev. 2016;74(12)7:723-736.
4. Tye C, Runicles AK, Whitehouse AJ, Alvares GA. Characterizing the interplay between autism spectrum disorder and comorbid medical conditions: An integrative review. Front Psychiatry. 2018;9:751.

5. Discroll A, Arnold LE, Williams K. Probiotics and autism spectrum disorder. In: $\mathrm{H}$ Razafindralambo (ed) Trends in Probiotic Applications. Studium Press LCC, Houston, USA, 2018;pp:171-184.

6. Hill C, Guarner F, Reid G. Expert consensus document: The international scientific association for probiotics and prebiotics consensus statement on the scope and appropriate use of the term probiotic. Nat Rev Gastroenterol Hepatol. 2014;11(8):506-514.

7. Tas AA. Dietary strategies in autism spectrum disorder (ASD). Progr Nutr. 2018;20:554-562.

8. Razafindralambo. Trends in probiotic applications. Studium Press LLC, Houston, USA, 2018.

9. Razafindralambo H, Rabetafika H, Razafindralambo A. Nutritionbased therapeutic approaches for solving autistic digestive troubles. Adv Food Technol Nutr Sci Open J. 2016;2(3):88-95.

10. Razafindralambo H, Razafindralambo A. Gluten intolerance as potential target for managing autism spectrum disorders: Towards innovative dietary approaches. Nov Tech Nutr Food Sci. 2019;3(2): $1-4$.

11. Zhang M, Ma W, Zhang J. Analysis of gut microbiota profiles and microbe-disease associations in children with autism spectrum disorders in China. Sci Rep. 2018;8:13981.

12. Kushak RI, Winter HS, Buie TM. Analysis of the duodenal microbiome in autistic individuals: Association with carbohydrate digestion. J Pediatr Gastroenterol Nutr. 2017;64(5):110-116.

13. Kulkarni S, Haq SF, Samant S. Adaptation of Lactobacillus acidophilus to thermal stress yields a thermotolerant variant which also exhibits improved survival at ph 2. Probiotics \& Antimicro Prot. 2018;10(4):717-727

14. Van der Mei HC, Bos R, Busscher HJ (1998) A reference guide to microbial cell surface hydrophobicity based on contact angles. Colloids Surf B Biointerfaces. 1998;11(4):213-221. 